\title{
Influence of Pulse Characteristics on Turbocharger Radial Turbine
}

Department of Engineering Mechanics, KTH Royal Institute of Technology, CCGEx,

Stockholm 10044, Sweden e-mail: rmosca@mech.kth.se

\section{Shyang Maw Lim}

Department of Engineering Mechanics, KTH Royal Institute of Technology, CCGEx,

Stockholm 10044, Sweden e-mail: smlim@mech.kth.se

Mihai Mihaescu

Department of Engineering Mechanics, KTH Royal Institute of Technology, CCGEx,

Stockholm 10044, Sweden e-mail: mihai@mech.kth.se
Turbocharging has been demonstrated as a key technology to enhance fuel efficiency in the automotive field that faces increasingly stringent emission regulations. Due to the reciprocating engine, pulsating flow feds the turbocharger turbine, which experiences conditions far from a continuous flow scenario. In this work, the effects of the characteristics of the mass flow pulse, parameterized through amplitude, frequency, and temporal gradient, are decoupled and studied via unsteady computational fluid dynamics calculations under on-engine operating conditions. First, the model is validated based on comparisons with experimental data in gas-stand conditions. Then, the effect of each parameter on the exergy budget is assessed by considering a $\pm 10 \%$ variation with respect to a baseline pulse. The other factors defining the operating conditions (e.g., mass flow, shaft speed, and inflow exergy) are kept the same as the baseline. The adopted approach enables to completely isolate the effects of each parameter in contrast with previous literature studies. Based on the results observed, pulse amplitude is identified as the primary parameter affecting the hot-side system response in terms of turbine performance, heat transfer, and entropy generation, while frequency and temporal gradient show a smaller influence compared to it. As the pulse amplitude increases, the turbine work is reported to improve up to $9.4 \%$. Smaller variations are otherwise observed for the frequency and temporal gradient analysis. With a $10 \%$ increase of the pulse frequency, the turbine work is registered to improve by $5.0 \%$, while the same percentage reduction of the temporal gradient leads to an increase of the turbine work equal to $3.6 \%$.

[DOI: 10.1115/1.4052498]

\section{Introduction}

With the increasingly stringent gas emission regulations, the need for higher energy-efficient internal combustion engines has never been a crucial topic as nowadays in the automotive field. The new regulations aim to inspire the research and development of new and more efficient technologies to fulfill the required standards. Among the circulated efficient solutions, air turbocharging has been demonstrated as an effective and mature technology in enhancing engine efficiency in conjunction with the socalled engine downsizing.

Under on-engine operating conditions, heat transfer and pulsating flow are characteristics of high importance to be considered with the turbocharger turbine. The radial turbine, essentially designed and optimized to operate under continuous flow [1], works in nonoptimal conditions, with deviations from gas-stand performance. The first experimental studies investigating pulsating flow effects on radial turbines date back to the mid- $60 \mathrm{~s}$ with the pioneering works by Wallace and Blair [2] and Benson and Scrimshaw [3], then followed by Benson [4], Kosuge et al. [5], Capobianco et al. [6], and Capobianco and Gambarotta [7]. However, it is only in 1986 with the work by Dale and Watson [8] that the hysteresis loop is reported for the first time in an experimental campaign at Imperial College. Over the pulse cycle, the turbine experiences deviations from the performance in gas-stand flow conditions, following a loop that is generally represented as mass flow parameter (MFP) against expansion ratio $(\pi)$, or isentropic efficiency $\left(\eta_{\text {is }}\right)$ against blade speed ratio. The area enclosed by the loop, a measure of the unsteadiness effects, is strongly dependent on the pulse characteristics, i.e., frequency and amplitude. Pulse frequency has received great attention from the authors as the primary parameter responsible for the unsteady behavior of the

\footnotetext{
${ }^{1}$ Corresponding author.

Manuscript received March 22, 2021; final manuscript received August 13, 2021 ; published online December 3, 2021. Assoc. Editor: Riccardo Da Soghe.
}

turbine. It is generally our knowledge that increasing the pulse frequency enlarges the hysteresis loop [9-16]. Based on this observation, different criteria have been proposed in the literature to determine whether the turbine operates as a quasi-steady device or not. Following Chen and Winterbone [17], who first made use of the Strouhal number, more complex formulations have been developed based on it. Szymko et al. [9] proposed a modified Strouhal number accounting for the pulse length fraction. Then, Copeland et al. [18] introduced a $\Lambda$ criterion to account for the pulse amplitude effects, subsequently redeveloped by Cao et al. [13] in order to include instantaneous pressure variation effects.

Besides frequency, pulse amplitude and shape profile affect the unsteady behavior of the turbine. It is reported in the literature that increasing pulse amplitude has the effect to enlarge the hysteresis loop [12-16,19]. This is motivated by the fact that the higher is the amplitude, the larger is the amount of mass flow which is found at a condition far from the cycle-averaged steady one. Recently, Lee et al. [20] and Rezk et al. [21] investigated the effects of different pulse shapes on turbine performance. The square profile was found to have the largest detrimental effect on the turbine efficiency compared to realistic, sinusoidal, and triangular profiles. A third pulse characteristic that is found to affect the unsteady behavior of the turbine is the temporal gradient. However, few efforts from the authors were dedicated to its characterization except for the work by Cao et al. [13], who reported an increase of the unsteadiness for a steeper front of the pressure wave.

In numerical studies investigating the effects of pulse amplitude and frequency, different pulse shapes are generally obtained by stretching or compressing the baseline pulse or the temporal window [13-16], respectively. A common approximation in nowadays literature is represented by the variation of the pulse temporal gradient together with the amplitude and frequency so that the pulse dynamics and evolution are different. If on one side this simplification is convenient, the major drawback is represented by the overlap of the temporal gradient effects with the 
amplitude and frequency ones. The approach followed in this work is different and aims to isolate the effects of every single characteristic by varying only the parameter of interest through a $\pm 10 \%$ deviation from the initial signal, while freezing the other parameters. The study investigates the effects of the pulse characteristics in terms of pulse amplitude, frequency, and temporal gradient, with the focus of defining an optimum criterion to maximize the turbine work by manipulating the pulse shape. This aspect is of great interest from an engine-turbocharger integrated system point of view, since amplitude and temporal gradient depend on the exhaust valve shape, while frequency on the engine regime. Moreover, an exergy-based post-processing approach is used to investigate the mechanisms underlying entropy generation and heat transfer. It is worth highlighting that the focus of this work is not to assess the deviation of the turbine performance between continuous and pulsating flow conditions, but to understand what parameters have beneficial effects on the turbine performance in order to optimize its working conditions. Furthermore, by following a different approach compared to previous authors, the pulse amplitude and frequency influence on the turbine performance is first decoupled from the temporal gradient effects.

\section{Methodology}

In this section, the computational method used is described and the sensitivity and validation studies are reported. The study investigates the performance of a single scroll 12-passage turbine system applied in a $2.0 \mathrm{~L}$ and four cylinders engine for passenger car. Since the aim is to replicate a model as close as possible to real on-engine conditions, the computational domain, represented in Fig. 1, incorporates the exhaust manifold, an important source of secondary flows in the volute [22]. The diffuser is followed by a convergent pipe with the outlet plane located at thirteen rotor diameters $(D=55 \mathrm{~mm})$ from the rotor-diffuser interface $\left(\mathcal{S}_{3}\right.$ with reference to Fig. 1$)$ in order to minimize the effects of the imposed boundary conditions.

2.1 Computational Setup. The governing equations, represented by the conservation of mass, momentum, and energy are solved through the commercial solver STAR-CCM+ by Siemens. Since the fluid is highly compressible and nonisothermal, a density-based coupled solver is chosen so that the governing equations are solved simultaneously. Air is used as working fluid, while density and pressure are linked together by the ideal gas law $(p=\rho \mathrm{RT})$. Furthermore, because of the large temperature variations, the molecular dynamic viscosity $\mu$ is modeled according to Sutherland's law, while the specific heat at constant pressure $c_{p}$ through a polynomial expression in the temperature. Unsteady Reynolds-averaged Navier-Stokes (RANS) are employed in conjunction with the two-equation $k-\omega \mathrm{SST}$ (shear stress transport) turbulence model [23] for both steady and pulsating flow simulations.

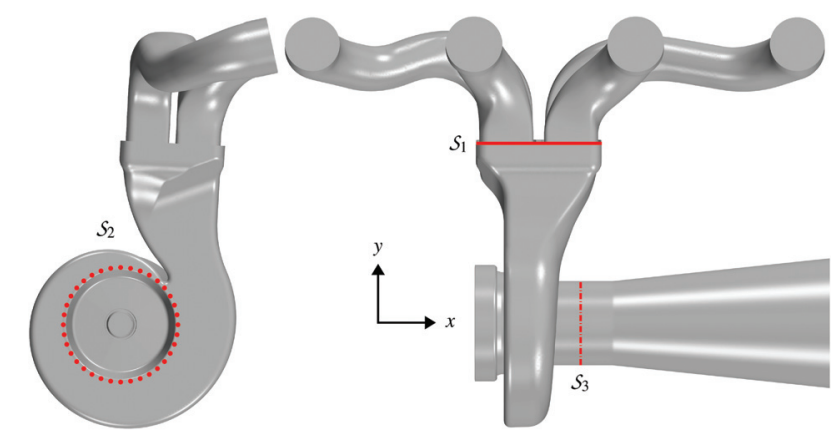

Fig. 1 Top ( $y z$ plane) and lateral ( $x y$ plane) views of the computational domain. Continuous line highlights the exhaust manifold-volute interface, $\mathcal{S}_{1}$, dotted line the volute-rotor interface, $\mathcal{S}_{2}$, and dashed line the rotor-diffuser interface, $\mathcal{S}_{3}$, respectively. The entire outlet pipe is not shown.
2.2 Boundary Conditions. A total of seven different cases are run in pulsating flow conditions. Starting from a baseline case, given by an experimentally calibrated GT-POWER model, the mass flow rate pulse is modified considering a $\pm 10 \%$ variation in amplitude, frequency, and temporal gradient. As the aim is to completely decouple the three pulse characteristics, the temporal gradient is kept constant for the amplitude and frequency studies. In addition, each case is characterized by the same cycle-averaged mass flow rate and inflow exergy, which is kept fixed by scaling the total temperature pulse. Details about the exergy model adopted can be found in Sec. 2.6.

In the numerical model, time-varying mass flow rate and total temperature are imposed at the exhaust manifold inlet. An entire engine cycle comprises four pulses, one for each runner, according to the firing sequence 2-1-3-4 (with reference to Fig. 1 and runners numbered from left to right). Turbulent inflow conditions are generated by a synthetic eddy method [24] with an imposed turbulent intensity equal to 5\% [25]. Walls are treated as smooth and diabatic, with two different constant temperatures adopted: the first, $T_{w 1}=1073 \mathrm{~K}$, is imposed at the exhaust manifold and volute walls, the second, $T_{w 2}=1001 \mathrm{~K}$, at the diffuser. Since the timescale associated with the solid material inertia is much larger than the pulse one [26], a constant temperature imposed at the wall represents a realistic condition. On contrary, rotor surfaces are treated as adiabatic, since the fraction of heat transfer occurring at the rotor is negligible compared to other parts of the system such as the volute and diffuser [27]. The rotor is physically rotated through the sliding mesh method with a constant rotational speed equal to 121,380 rpm and kept fixed for each pulsating flow case. At the outlet boundary, time-varying static pressure and temperature are given.

2.3 Mesh Sensitivity Under Pulsating Flow. As the flow characteristics under on-engine conditions largely differ from the ones in steady flow, a mesh sensitivity study is carried under pulsating flow by considering the baseline pulse (subsequently reported in Sec. 3). As consequence of the large velocity and temperature gradients, the evaluation of the turbine torque and heat transfer needs special attention in terms of wall resolution. For this reason, four different grids with increasing wall resolution are tested. The results of the analysis are summarized in Table 1, while time-varying turbine torque and heat transfer rate at the exhaust manifold are reported in Figs. 2 and 3, respectively. The data show a small $(\approx+2 \%)$ percentage difference of the cycle-averaged turbine torque between G4 (fine) and G1 (coarse), mainly located at the pulse peak, which is slightly underpredicted for G1 (see Fig. 2). Heat transfer rate shows otherwise a slower convergence as reported in Fig. 3. Indeed, comparing G4 and G1, the differences in the pulse peak and cycle-averaged values are $\approx-9.0 \%$ so that mesh independence is reached starting from G3. As consequence, in the final setup the wall resolution adopted in G2 is used at the rotor surface while G3 resolution over the stationary surfaces.

The final mesh is constituted by $22 \mathrm{M}$ polyhedral elements distributed as in the following: $10 \%$ in the exhaust manifold, $32 \%$ in the volute, $35 \%$ in the rotor, and $21 \%$ in the diffuser. The remaining $2 \%$ is distributed in the outlet convergent duct, where the grid is

Table 1 Cycle-averaged heat transfer rate $\dot{Q}$ at the exhaust manifold, turbine torque $\tau$, and maximum surface-averaged $y_{+}$ for increasing grid resolution

\begin{tabular}{lccccc}
\hline \hline & \multicolumn{2}{c}{ Exhaust manifold } & & \multicolumn{2}{c}{ Rotor } \\
\cline { 2 - 3 } \cline { 5 - 6 } & $\dot{Q}_{\text {man }}(\mathrm{kW})$ & $y_{+, \max }$ & & $\tau(\mathrm{Nm})$ & $y_{+, \max }$ \\
\hline G1 & 2.65 & 13.0 & & 1.07 & 9.88 \\
G2 & 2.30 & 1.32 & & 1.10 & 2.46 \\
G3 & 2.42 & 0.62 & & 1.09 & 0.56 \\
G4 & 2.41 & 0.48 & & 1.09 & 0.42 \\
\hline \hline
\end{tabular}




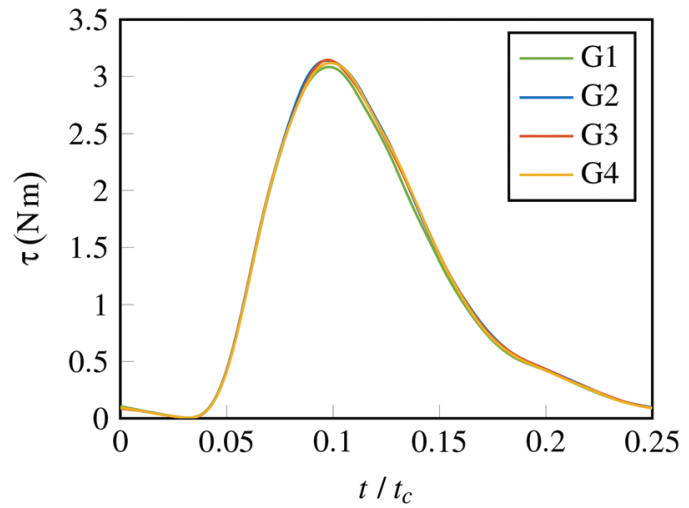

Fig. 2 Instantaneous turbine torque against normalized time over one pulse cycle

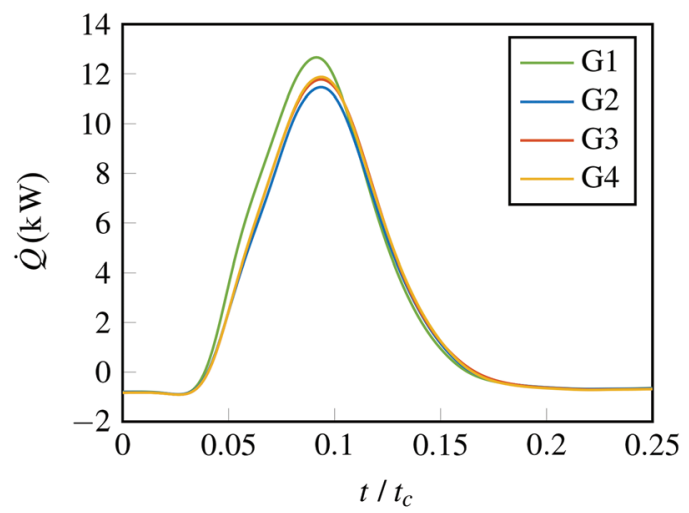

Fig. 3 Instantaneous heat transfer rate at the exhaust manifold against normalized time over one pulse cycle

gradually stretched. The maximum surface-averaged $y_{+}$is kept close to unity to avoid the use of scalable wall functions (see Table 1).

2.4 Validation Under Steady Flow. The model is validated against experimental data obtained in gas-stand flow experiments. At first, for the evaluation, the exhaust manifold is removed from the computational domain and replaced by a straight pipe $310 \mathrm{~mm}$ long. Then, a steady-state simulation is performed through the use of a multireference frame method. The walls are treated as adiabatic since, under continuous flow conditions, the expansion ratio has been demonstrated to not vary with respect to the type of boundary condition imposed [28].

Figure 4 shows the turbine performance map as mass flow parameter

$$
\mathrm{MFP}=\frac{\dot{m} \sqrt{T_{t}}}{p_{t}}
$$

with quantities calculated at the exhaust manifold-volute interface $\left(\mathcal{S}_{1}\right.$, with reference to Fig. 1$)$, against the expansion ratio

$$
\pi=\frac{p_{t, \text { in }}}{p_{\text {out }}}
$$

with the outlet static pressure, $p_{\text {out }}$, calculated at the outlet plane and equal to the atmospheric pressure. The data in Fig. 4 show a good agreement between experimental data and numerical results, demonstrating a good reliability of the model. Percentage differences, below $3 \%$, are probably due to unavoidable differences between experimental and numerical set-ups such as measurement locations, uncertainty in the boundary conditions, or geometry imperfections.

2.5 Time-Step Sensitivity. A time-step sensitivity study has been performed using G1 to assess the turbine torque and heat

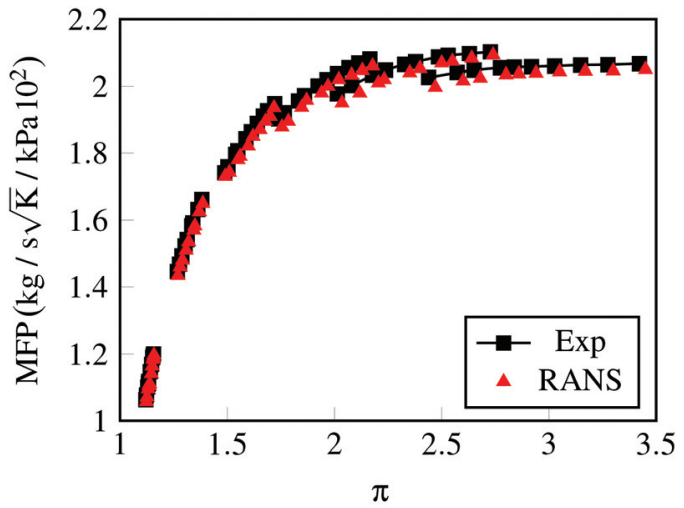

Fig. 4 Turbine performance map as MFP against expansion ratio $(\pi)$

transfer sensitivity to the temporal discretization under pulsating flow conditions. Three different time-steps, corresponding to $0.5 \mathrm{deg}, 1 \mathrm{deg}$, and $3 \mathrm{deg}$ of rotation of the rotor, were considered. Since the investigation has highlighted no significant difference, where variations have been found smaller than $1 \%$, the time-step corresponding to $3 \mathrm{deg}$ of rotation of the rotor is chosen to reduce the computational cost of the final setup.

2.6 Post-Processing. In the post-processing phase, an exergy-based analysis is employed to investigate the global system performance. The reader is referred to the existing literature for the application of the below-mentioned flow exergy methodology in turbomachinery applications (e.g., see Refs. [22,29,30]).

For a general open system, the specific exergy (in the reference frame of a given environment) is the maximum amount of shaft work per unit mass that can be extracted when the considered system is at thermal and mechanical equilibrium with the environment, i.e., the dead state. Mathematically, the specific exergy is expressed as

$$
e_{f}=h_{t}\left(T_{t}\right)-h_{o}-T_{o}\left[s\left(T_{t}, p_{t}\right)-s_{o}\right]
$$

where $h$ and $s$ represent the specific enthalpy and entropy, respectively, while the subscripts $t$ and $o$ denote the total quantity and the dead state. Here, the dead state is chosen as the ambient conditions $\left(T_{o}=298.15 \mathrm{~K}, p_{o}=101.3 \mathrm{kPa}\right)$ so that an evaluation of the theoretical maximum shaft work that could be extracted from the global system perspective is possible.

During an irreversible process, exergy is always destroyed [31]. By accounting for all the exergy streams in and out of the system, the exergy destruction (or internal exergy loss) is proportional to the entropy generation and can be determined using the exergy budget equation which, in integral form, reads as

$$
\begin{aligned}
& \underbrace{\frac{d}{d t}\left[\iint_{V^{*}(t)}\left(\rho e_{f}\right) \mathrm{d} V\right]}_{A}+\underbrace{\oiint_{S^{*}(t)}\left[\rho e_{f}\left\{\left(\mathbf{u}-\mathbf{u}_{b}\right) \cdot \mathbf{n}\right\}\right] \mathrm{d} S}_{-B} \\
& =-\underbrace{\oiint_{S^{*}(t)}\left[\left(\mathbf{r} \times \mathbf{f}_{\text {net }}\right) \cdot \mathbf{\Omega}\right] \mathrm{d} S}_{C}-\underbrace{\oiint_{S^{*}(t)}\left[\left(1-\frac{T_{o}}{T_{w}}\right)(\mathbf{q} \cdot \mathbf{n})\right] \mathrm{d} S}_{D} \\
& -\underbrace{T_{o} \dot{S}_{\text {gen }}}_{E}+\underbrace{\frac{d}{d t}\left[\iiint_{V^{*}(t)} p \mathrm{~d} V\right]}_{F}
\end{aligned}
$$

Here, $\mathbf{u}$ is the velocity of a Lagrangian fluid particle, and $\mathbf{u}_{b}$ is the local velocity of the control surface $S^{*}(t)$ enclosing an arbitrary moving control volume $V^{*}(t)$. The outward normal vector of $S^{*}(t)$ 
is depicted by $\mathbf{n} . \mathbf{f}_{\text {net }}, \mathbf{r}, \boldsymbol{\Omega}$ and $\mathbf{q}$ represent the net force on the rotor surface per unit area vector, position vector, rotational speed vector, and heat flux vector, respectively. Exergy can be transferred and destroyed through different physical mechanisms which, with reference to Eq. (4), are listed below:

- A: local temporal rate of change of the flow exergy in $V^{*}(t)$

- $B$ : net flow exergy change due to advection of the fluid particles through $S^{*}(t)$

- $C$ : shaft power $\dot{W}_{T}$ across $S^{*}(t)$

- $D$ : flow exergy transfer via heat transport across $S^{*}(t)$

- E: flow exergy loss via total internal irreversibilities in $V^{*}(t)$

- $F$ : unsteady contribution in $V^{*}(t)$

The determination of the exergy losses via total internal irreversibilities (term $E$ ) can be obtained by rearranging Eq. (4) as

$$
E=-B-C-D-(A-F)
$$

and by computing the different terms of the RHS of Eq. (5) numerically. However, this approach does not provide any information on the nature of the internal losses which, in a thermofluidic system as the one taken into consideration in this work, are caused by both viscous and thermal processes. If the relative importance of the aerothermodynamic losses is of interest, the total internal irreversibilities can be computed by volume integration of the viscous and thermal entropy generation rate. The first is given by

$$
\dot{S}_{\text {gen }, \text { viscous }}=\iiint_{V^{*}(t)}\left[\frac{\mu_{\text {eff }}}{T}\left(2 S_{i j} S_{i j}-\frac{2}{3} S_{k k} S_{k k}\right)\right] \mathrm{d} V
$$

where $S_{i j}$ is the strain rate tensor, i.e., the symmetric part of the velocity gradient tensor $\partial u_{i} / \partial x_{j}$, and $\mu_{\text {eff }}$ is the effective dynamic viscosity, sum of the molecular and turbulent ones. The latter reads as

$$
\dot{S}_{\text {gen,thermal }}=\iiint_{V^{*}(t)}\left[\frac{k_{\mathrm{eff}}}{T^{2}}\left(\frac{\partial T}{\partial x_{j}}\right)^{2}\right] \mathrm{d} V
$$

with $k_{\text {eff }}$ the effective thermal conductivity, sum of the molecular and turbulent ones.

The two proposed approaches for computing entropy generation are named indirect and direct method [32], respectively. Despite the direct method enables the characterization of the different types of physics mechanisms contributing to the entropy generation, it is more resource demanding, since it involves volume integration of the velocity and temperature gradients. As consequence, the direct method is particularly sensitive to the spatial discretization as demonstrated by Lim et al. [29] in a comparison of the two methods in turbomachinery applications. In this work, the direct method is adopted, since the assessment of the aerothermodynamic losses is of interest. To account for the relative importance of viscous and thermal irreversibilities, the Bejan number [33]

$$
\mathrm{Be}=\frac{1}{1+\frac{\dot{S}_{\text {gen,viscous }}}{\dot{S}_{\text {gen,thermal }}}}
$$

is considered, with the ratio $\dot{S}_{\text {gen,viscous }} / \dot{S}_{\text {gen,thermal }}$ representing the so-called irreversibility factor. Bejan number ranges from 0 to 1 , depending on whether the entropy generation is dominated by viscous or thermal mechanisms, respectively.

One factor of uncertainty in pulse parametric studies derives from the different theoretical maximum work achievable at the turbine as pulse shape changes. This aspect limits the ability to generalize trends of previous literature studies, since the turbine performance is inherently influenced by the theoretical maximum work achievable. In this work, to overtake the limitation, a constraint on the cycle-averaged inflow exergy has been imposed during the pulse design. For each pulsating flow case considered, in order to guarantee complete comparability, the cycle-averaged inflow exergy $\dot{m} e_{f}$ is kept constant by scaling the inlet total temperature pulse. As consequence, the system response is not affected by a different exergetic content, i.e., the theoretical maximum work achievable. Furthermore, this procedure not only represents a stricter methodology compared to previous pulse characteristics studies, but also enables a direct comparison among the different pulse characteristics.

\section{Results}

In this section, the results of the pulse characteristics study are presented and analyzed. Simulations are run for a total of two engine cycles at an engine regime point equal to $2400 \mathrm{rpm}$. Statistics are calculated by excluding the first cycle in order to ensure proper initialization of the flow field. For purpose of clearness, the time-varying plots show data relative to one single pulse, while statistics are calculated over the entire engine cycle. In the following, percentage differences are meant to be with respect to the baseline case so that, for a given general quantity $\phi$, variations are reported as $\Delta \phi=\left(\phi-\phi_{\text {baseline }}\right) / \phi_{\text {baseline }}$.

3.1 Pulse Amplitude Effects. The first parameter studied is the pulse amplitude, which has been extensively demonstrated to be one major source of unsteadiness by different authors [13-16]. The mass flow rate and total temperature pulses imposed as inlet boundary conditions are represented in Fig. 5. The increase in mass flow rate at the pulse peak is compensated by lower values in the intra-pulse phase (see Fig. $5(a)$ ) in order to enforce the same cycle-averaged mass flow rate among pulses. The total temperature profile (see Fig. 5(b)) is uniformly scaled in order to guarantee a constant cycle-averaged inflow exergy in the system.

The exergy budget and Bejan number are depicted in Fig. 6 for the three different cases with reference to the single components of the domain: exhaust manifold, volute, and rotor. Increasing the pulse amplitude shows beneficial effects on the turbine performance. Indeed, the turbine work percentage variations for the $0.9 \mathrm{~A}$ and $1.1 \mathrm{~A}$ cases (with respect the baseline one) are reported equal to $-4.2 \%$ and $+9.4 \%$ (see Fig. 6(a) and Table 2). A deeper understanding of this trend is given by the instantaneous expansion ratio represented in Fig. 7. The impact of the pulse amplitude is remarkable at the pulse peak, where the differences between the baseline case with the $0.9 \mathrm{~A}$ and $1.1 \mathrm{~A}$ cases are equal to $-9.3 \%$ and $+11.0 \%$, respectively. Smaller variations are otherwise registered for the cycle-averaged expansion ratio, equal to $1.79,1.83$, and 1.90 for each case, respectively. The data show that increasing the pulse amplitude enhances both the instantaneous and the cycle-average expansion capacity of the turbine. As the pulse amplitude increases, the higher expansion ratio values in the pulse phase compensate the lower ones occurring in the intra-pulse phase so that the overall effect results in a higher cycle-averaged expansion ratio and turbine torque (see Table 2).

The growth of the power output with increasing pulse amplitude was already observed by Zhao et al. [34] on the high pressure turbine of a two-stage turbocharger system together with a drop of the turbine efficiency. This apparent inconsistency is related to the larger theoretical isentropic expansion power available at the turbine as the pulse amplitude increases. This aspect, as previously discussed, limits the capacity of generalization of the trends of the turbine performance with respect to variations of the pulse characteristics. Indeed, it is difficult to relate the variation of the turbine performance to the specific pulse parameter rather than the maximum theoretical work achievable. In the present case, both turbine power and efficiency improve with increasing pulse amplitude. By considering the so-called exergy utilization, defined as the fraction of inflow exergy converted into mechanical power 


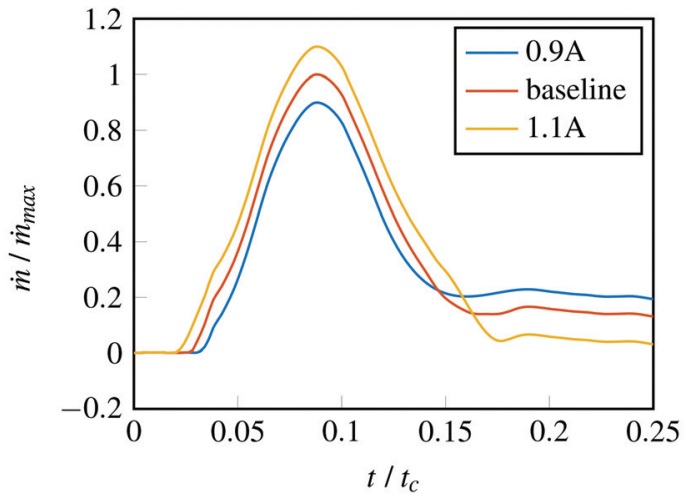

(a)

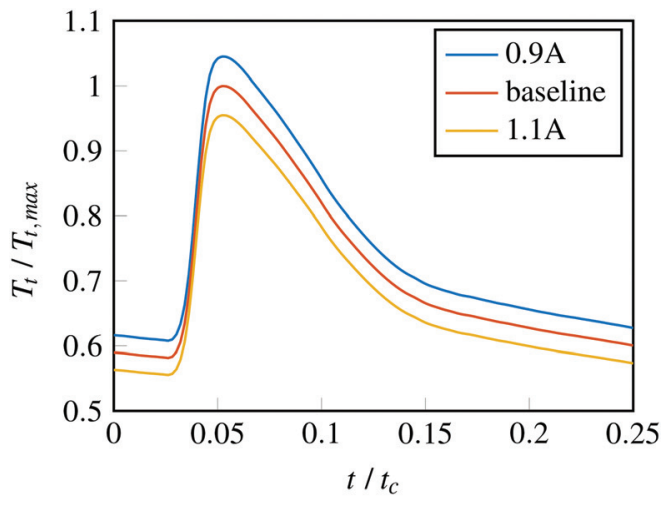

(b)

Fig. 5 Boundary conditions imposed at the exhaust manifold inlet in case of the pulse amplitude study. The axis are normalized by the engine period $t_{c}=0.0499 \mathrm{~s}$, the baseline maximum mass flow rate $\dot{m}_{\max }=0.295 \mathrm{~kg} / \mathrm{s}$, and the baseline maximum total temperature $T_{t, \max }=1639 \mathrm{~K}$, respectively. Only one pulse over four is visualized. (a) Time-varying mass flow rate. (b) Time-varying total temperature.

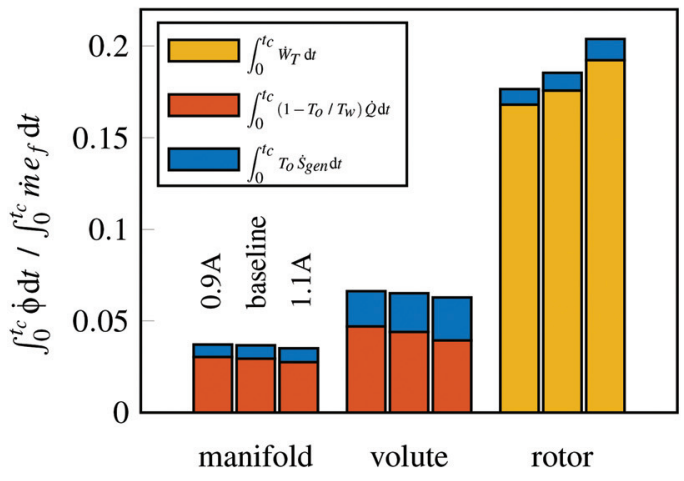

(a)

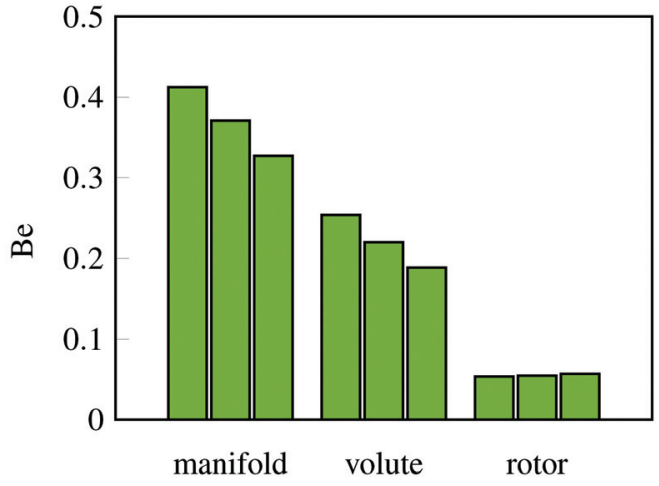

(b)

Fig. 6 Pulse amplitude effects on exergy budget and Bejan number with reference to the different system components: exhaust manifold, volute, and rotor. Turbine work and exergy loss via heat transfer and total internal irreversibilities are visualized in the exergy budget normalized by the cycle-averaged inflow exergy $\dot{m} e_{f}$. If entropy generation rate is dominated by viscous mechanisms over thermal ones, $\mathrm{Be} \rightarrow 0$, vice versa, $\mathrm{Be} \rightarrow 1$. (a) Exergy budget. (b) Bejan number.

Table 2 Pulse amplitude study quantitative data for the time integral of turbine power (term $C$ ), flow exergy loss via heat transfer (term $D$ ), and total internal irreversibilities (term $E$ with reference to Eq. (4)) with viscous and thermal contributions highlighted. Cycle-averaged and maximum temperatures are calculated at the inlet volute section $\left(\mathcal{S}_{1}\right)$. Cycle-averaged and maximum turbine torque are also reported.

\begin{tabular}{|c|c|c|c|c|c|c|c|c|c|}
\hline & \multicolumn{3}{|c|}{ Exergy budget } & \multicolumn{2}{|c|}{ Internal irreversibilities } & \multicolumn{2}{|c|}{ Temperature } & \multicolumn{2}{|c|}{ Torque } \\
\hline & $C(\mathrm{~J})$ & $D(\mathrm{~J})$ & $E(\mathrm{~J})$ & $T_{o} S_{\text {gen,viscous }}(\mathrm{J})$ & $T_{o} S_{\text {gen,thermal }}(\mathrm{J})$ & $T_{\mathcal{S}_{2}, \text { avg }}(\mathrm{K})$ & $T_{\mathcal{S}_{2}, \max }(\mathrm{K})$ & $\tau_{\text {avg }}(\mathrm{Nm})$ & $\tau_{\max }(\mathrm{Nm})$ \\
\hline $0.9 \mathrm{~A}$ & 667 & 306 & 137 & 105 & 32 & 1171 & 1482 & 1.05 & 2.83 \\
\hline Baseline & 696 & 291 & 151 & 120 & 31 & 1139 & 1447 & 1.10 & 3.18 \\
\hline $1.1 \mathrm{~A}$ & 762 & 266 & 168 & 138 & 30 & 1098 & 1388 & 1.20 & 3.56 \\
\hline
\end{tabular}

$\left(\eta_{\mathrm{ex}}=\dot{W}_{T} / \dot{m} e_{f}\right)$, its increase is driven only by the growth of the turbine work, since the cycle-averaged inflow exergy is the same if calculated at the exhaust manifold inlet. This consideration shows the importance of defining boundary conditions that are fully comparable in terms of theoretical work achievable in the system, as done with the exergy-based method used in this work.

With increasing amplitude, the exergy lost by heat transfer is characterized by a monotonic descending trend, both into the exhaust manifold and volute. Percentage variations are reported to be equal to $+5.1 \%$ and $-8.6 \%$ for the $0.9 \mathrm{~A}$ and $1.1 \mathrm{~A}$ cases, respectively. This result is strongly influenced by the scaling of the total temperature profiles (see Fig. 5(b)) employed to match the cycle-averaged inflow exergy. Consequently, the larger amplitude cases are characterized by smaller cycle-averaged and maximum temperatures at the exhaust manifold, so that the inlet temperature at the volute inlet is higher for the $0.9 \mathrm{~A}$ case (see Table 2). Heat transfer is otherwise null in the rotor because of the imposition of adiabatic boundary condition at the wall.

The exergy losses via total internal irreversibilities, sum of viscous and thermal ones, experience an ascending trend with increasing pulse amplitude, with variations equal to $-9.3 \%$ and $+11.2 \%$ for the $0.9 \mathrm{~A}$ and $1.1 \mathrm{~A}$ cases, respectively. A higher 


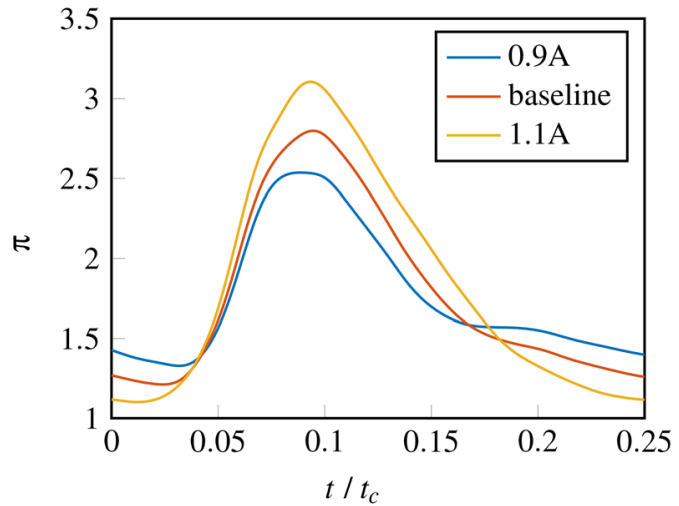

Fig. 7 Instantaneous expansion ratio against normalized time

entropy generation is caused by the increase of the viscous irreversibilities for a larger pulse amplitude, which is related to larger velocity gradients (see Table 2). This trend is well reflected in Fig. 6(b), where the Bejan number monotonically decreases with increasing pulse amplitude in both the exhaust manifold and volute It is also worth noticing that $\approx 55 \%$ of the total internal irreversibilities are generated inside the volute. In the rotor, entropy generation is otherwise dominated by viscous mechanisms both because of the adiabatic boundary condition imposed at the rotor surface and the large velocity gradients caused by the rotational motion of the turbine.

3.2 Pulse Frequency Effects. The second parameter studied is frequency. The procedure followed for the analysis of the pulse frequency effects differs from the one generally adopted in the literature, where different pulses are obtained by scaling the temporal window [11-16]. Although this procedure is simple, it has the collateral effect of modifying the pulse shape, in particular the temporal gradient of the pulse, resulting in the overlap of the two characteristics effects. In this work, the procedure followed aims to completely decouple the frequency effects from the temporal gradient, which is kept constant together with the pulse amplitude. As consequence of the methodology adopted, the analysis tends to highlight the effects of the intra-pulse phase, since the shape of the pulse is maintained fixed as reported in Fig. 8, where the timevarying mass flow rate is shown together with the total temperature imposed as boundary conditions. It is also worth highlighting that the mass flow rate at the end tail of the pulse is modified in order to enforce a constant cycle-averaged mass flow rate among pulses.

The exergy budget, Fig. 9(a), highlights the beneficial effect of increasing frequency on the turbine work with variations equal to $-1.6 \%$ and $+5.0 \%$ with respect to the baseline case (see also Table 3). Although the turbine response to pulse frequency is

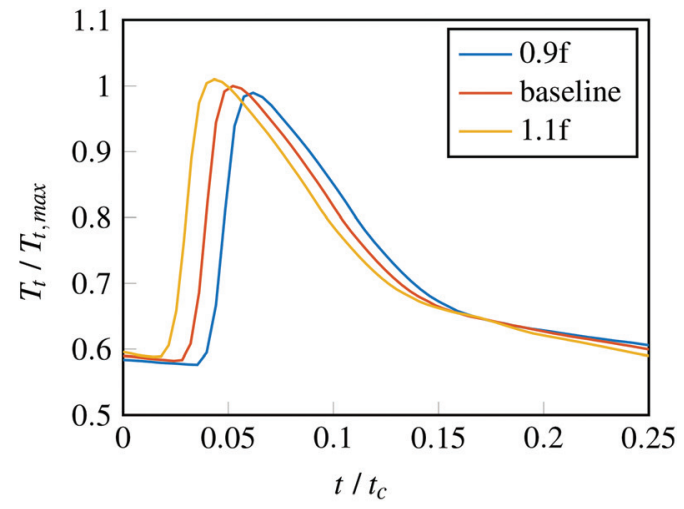

(b)

Fig. 8 Boundary conditions imposed at the exhaust manifold inlet in case of the pulse frequency study. The axis are normalized by the engine period $t_{c}=0.0499 \mathrm{~s}$, the baseline maximum mass flow rate $\dot{m}_{\max }=0.295 \mathrm{~kg} / \mathrm{s}$, and the baseline maximum total temperature $T_{t, \max }=1639 \mathrm{~K}$, respectively. Only one pulse over four is visualized. (a) Time-varying mass flow rate. (b) Time-varying total temperature.

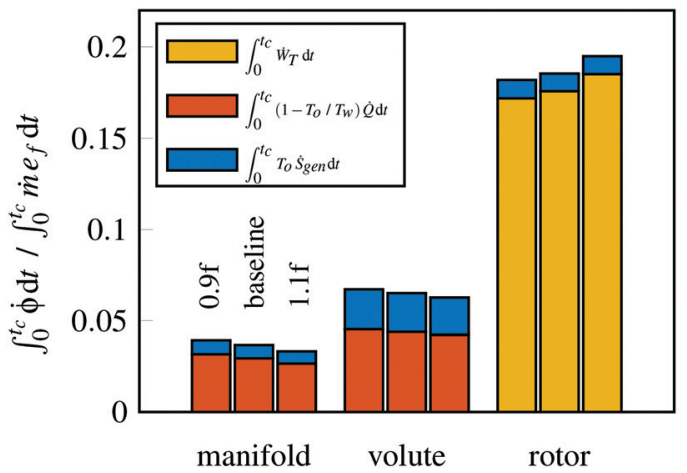

(a)

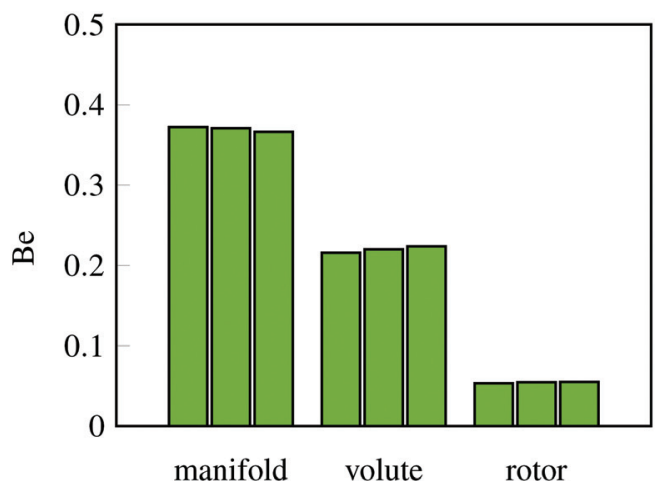

(b)

Fig. 9 Pulse frequency effects on exergy budget and Bejan number with reference to the different system components: exhaust manifold, volute, and rotor. Turbine work and exergy loss via heat transfer and total internal irreversibilities are visualized in the exergy budget normalized by the cycle-averaged inflow exergy $\dot{m} e_{f}$. If entropy generation rate is dominated by viscous mechanisms over thermal ones, $\mathrm{Be} \rightarrow 0$, vice versa, $\mathrm{Be} \rightarrow$ 1. (a) Exergy budget. (b) Bejan number. 
Table 3 Pulse frequency study quantitative data for the time integral of turbine power (term $C$ ), flow exergy loss via heat transfer (term $D$ ), and total internal irreversibilities (term $E$ with reference to Eq. (4)) with viscous and thermal contributions highlighted. Cycle-averaged and maximum temperatures are calculated at the inlet volute section $\left(\mathcal{S}_{1}\right)$. Cycle-averaged and maximum turbine torque are also reported.

\begin{tabular}{|c|c|c|c|c|c|c|c|c|c|}
\hline & \multicolumn{3}{|c|}{ Exergy budget } & \multicolumn{2}{|c|}{ Internal irreversibilities } & \multicolumn{2}{|c|}{ Temperature } & \multicolumn{2}{|c|}{ Torque } \\
\hline & $C(\mathrm{~J})$ & $D(\mathrm{~J})$ & $E(\mathrm{~J})$ & $T_{o} S_{\text {gen,viscous }}(\mathrm{J})$ & $T_{o} S_{\text {gen,thermal }}(\mathrm{J})$ & $T_{\mathcal{S}_{2}, \text { avg }}(\mathrm{K})$ & $T_{\mathcal{S}_{2}, \max }(\mathrm{K})$ & $\tau_{\text {avg }}(\mathrm{Nm})$ & $\tau_{\max }(\mathrm{Nm})$ \\
\hline $0.9 \mathrm{f}$ & 685 & 307 & 158 & 126 & 32 & 1130 & 1440 & 0.98 & 3.14 \\
\hline Baseline & 696 & 291 & 151 & 120 & 31 & 1139 & 1439 & 1.10 & 3.18 \\
\hline $1.1 \mathrm{f}$ & 731 & 272 & 145 & 115 & 30 & 1147 & 1440 & 1.28 & 3.28 \\
\hline
\end{tabular}

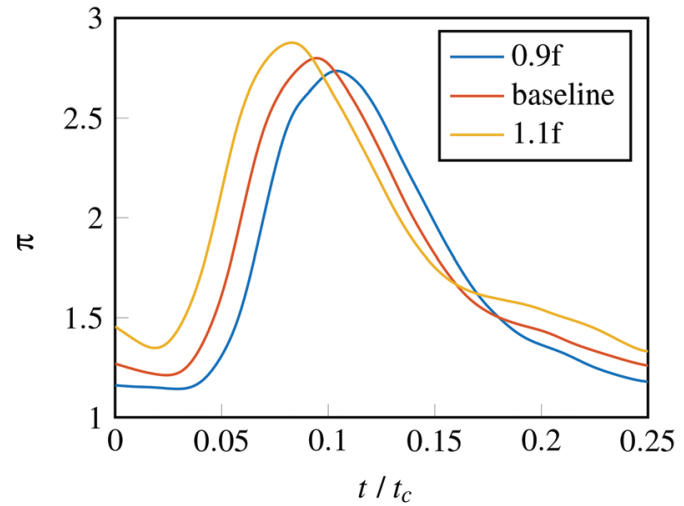

Fig. 10 Instantaneous expansion ratio against normalized time

smaller compared to the amplitude, frequency still has a significant influence on both cycle-averaged and instantaneous turbine performance. Indeed, despite the pulse shape is kept fixed, remarkable variations can be observed in the instantaneous expansion ratio in Fig. 10, where the percentage differences at the pulse peak, equal to $-2.3 \%$ and $+2.8 \%$ for the $0.9 f$ and $1.1 \mathrm{f}$ cases, are significant. Higher expansion ratio values are found also during the intra-pulse phase for increasing frequency, mainly because the mass flow rate is higher in this phase in order to satisfy the mass flow constraint for the boundary conditions (see Fig. 8(a)). The combination of the two effects is beneficial for the cycle-averaged turbine torque, which is higher for the $1.1 \mathrm{f}$ case compared to the 1.1A case (compare Tables 2 and 3 ).

As for the turbine cycle-averaged and instantaneous behavior, the effect of frequency on heat transfer is significant, especially at the exhaust manifold, and comparable to the pulse amplitude.
Percentage variations are registered equal to $+5.5 \%$ and $-6.5 \%$ for the $0.9 \mathrm{f}$ and $1.1 \mathrm{f}$ cases, respectively, so that heat transfer is subject to a descending trend with increasing pulse frequency. The effect of the scaling of the total temperature profile is negligible at the pulse peak; indeed, the maximum temperature at the volute is the same for all the three cases considered as reported in Table 3. As consequence, the instantaneous heat transfer rate has a similar trend for all three cases during the pulse phase, while a different behavior is registered during the intra-pulse phase, as expected. Here, the mass flow rate and temperature are low so that the heat transfer occurs from the surrounding to the domain. The reverse heat transfer is larger with increasing pulse frequency, since the minimum temperature at the volute inlet is registered to decrease and equal to $307 \mathrm{~K}, 291 \mathrm{~K}$ and $272 \mathrm{~K}$ for the 0.9f, baseline, and $1.1 \mathrm{f}$ cases, respectively. It is worth noticing that the cycle-averaged temperature at the volute inlet increases with increasing pulse frequency (see Table 3). This is justified by the fact that the intrapulse phase, characterized by lower temperatures, has a shorter duration for the $1.1 \mathrm{f}$ case, so that the cycle-average temperature increases.

The quantification of the entropy generation mechanisms shows a smaller impact of the frequency on the total internal irreversibilities compared to the amplitude study. Exergy losses via both viscous and thermal processes decrease similarly with increasing frequency due to the shorter cycle duration. As a consequence, the Bejan number (see Fig. 9(b)) displays small variations in the different components of the system.

3.3 Temporal Gradient Effects. The final parameter taken into consideration is the temporal gradient, which is arbitrarily calculated as the derivative between the pulse peak, kept constant, and the instant corresponding to the normalized mass flow rate equal to 0.05 (see Fig. 11(a)). Kosuke et al. [5] and Copeland et al. [18] first addressed the temporal gradient of the pulse wave

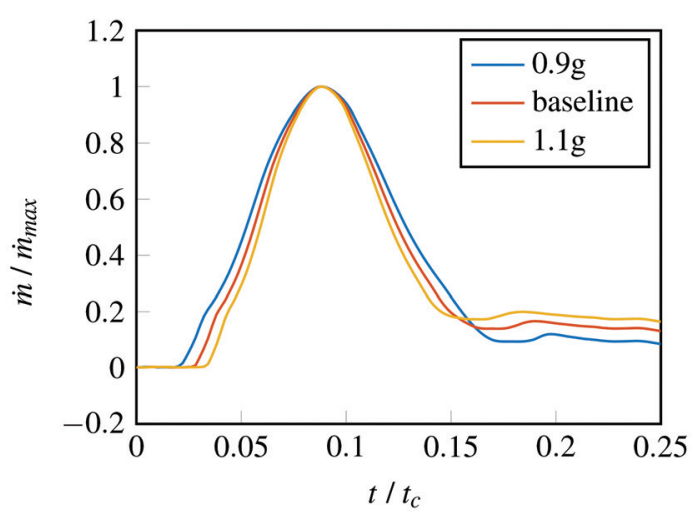

(a)

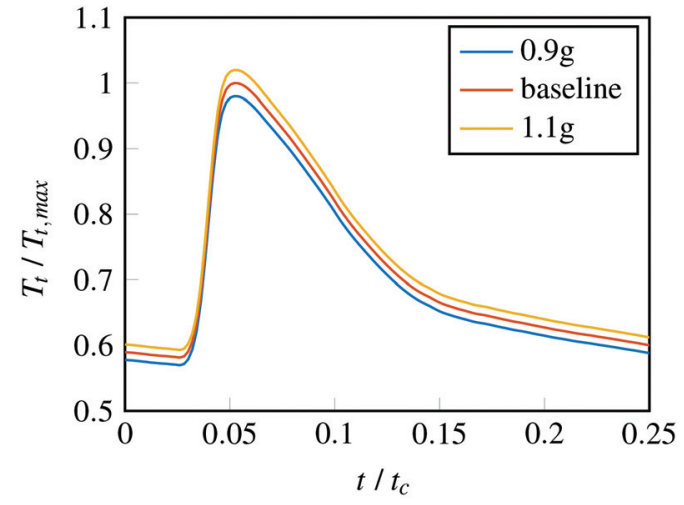

(b)

Fig. 11 Boundary conditions imposed at the exhaust manifold inlet in case of the temporal gradient study. The axis are normalized by the engine period $t_{c}=0.0499 \mathrm{~s}$, the baseline maximum mass flow rate $\dot{m}_{\max }=0.295 \mathrm{~kg} / \mathrm{s}$, and the baseline maximum total temperature $T_{t, \max }=1639 \mathrm{~K}$, respectively. Only one pulse over four is visualized. (a) Time-varying mass flow rate. (b) Time-varying total temperature. 


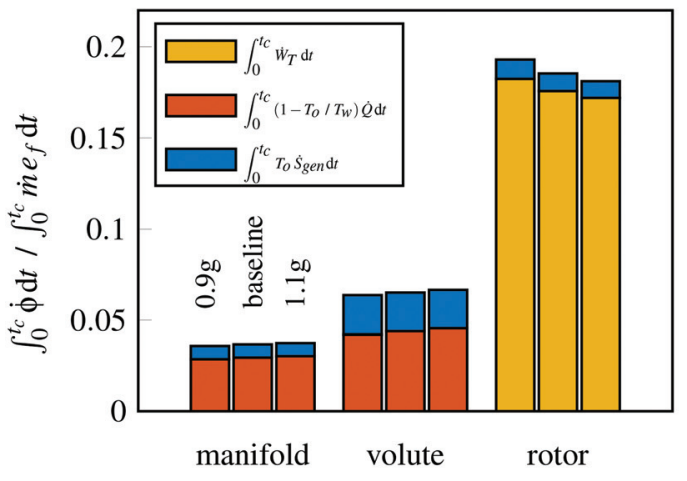

(a)

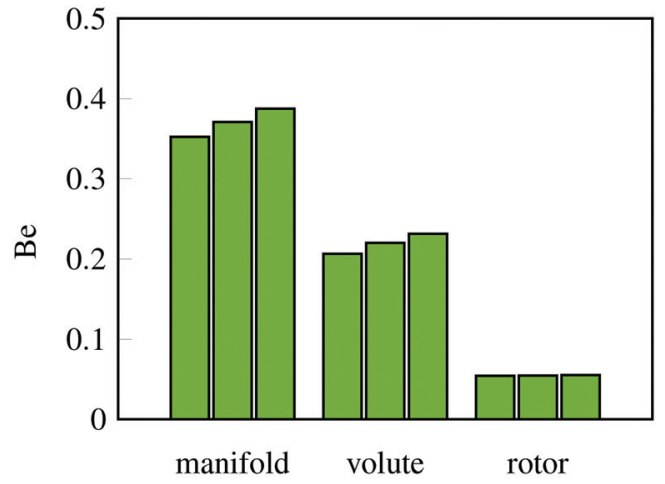

(b)

Fig. 12 Temporal gradient effects on exergy budget and Bejan number with reference to the different system components: exhaust manifold, volute, and rotor. Turbine work and exergy loss via heat transfer and total internal irreversibilities are visualized in the exergy budget normalized by the cycle-averaged inflow exergy $\dot{m} e_{f}$. If entropy generation rate is dominated by viscous mechanisms over thermal ones, $\mathrm{Be} \rightarrow 0$, vice versa, $\mathrm{Be} \rightarrow 1$. (a) Exergy budget. (b) Bejan number.

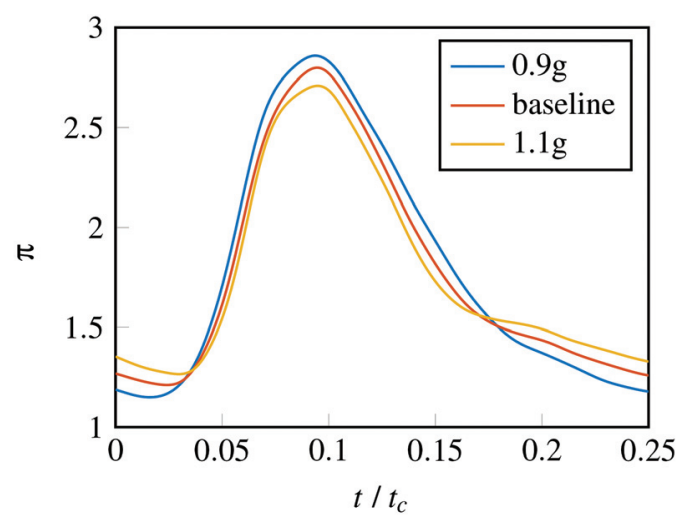

Fig. 13 Instantaneous expansion ratio against normalized time

as a contributor in the amplitude and frequency studies without any specific investigation on it. Then, in a numerical study, Cao et al. [13] highlighted the importance of the pressure wave front gradient as a source of unsteadiness for the turbine performance. Here, its effect is shown in the exergy budget (see Fig. 12(a)) with the turbine work decreasing monotonically for a steeper temporal gradient. The percentage differences for the $0.9 \mathrm{~g}$ and $1.1 \mathrm{~g}$ cases with respect to the baseline pulse are equal to $+3.6 \%$ and $-2.7 \%$, respectively, showing a remarkable influence of the temporal gradient on the turbine performance. An explanation of this trend is given by the instantaneous expansion ratio represented in Fig. 13: a slower acceleration of the flow gives rise to a higher expansion peak that is related to a larger mass accumulation in the volute during the pulse phase. As consequence, the mass flow rate pulse peak at the volute inlet is higher for the lower temporal gradient case so that the effect is similar to the one produced by increasing the pulse amplitude, but less severe. For the $0.9 \mathrm{~g}$ case, similarly to what reported for the $1.1 \mathrm{~A}$ case, the lower expansion ratio values produced during the intra-pulse phase are compensated by larger values in the pulse phase, with the overall effect of increasing the turbine work. Furthermore, both the cycle-averaged and maximum torque decrease with increasing temporal gradient (see Table 4) demonstrating a significant effect of the temporal gradient both on the instantaneous and cycle-averaged quantities.

The impact of the temporal gradient on heat transfer, which experiences a monotonic ascending trend with increasing temporal gradient, is weaker compared to pulse amplitude and frequency. The percentage differences for the $0.9 \mathrm{~g}$ and $1.1 \mathrm{~g}$ cases, equal to $-4.1 \%$ and $+3.4 \%$, respectively, are explained by the smaller cycle-averaged and maximum temperatures registered at the volute inlet as result of the scaling of the total temperature profile (see Table 4). As consequence, the heat transfer rate peak is higher for increasing temporal gradient.

Compared to the amplitude and frequency, total internal irreversibilities show a limited response to temporal gradient changes with variations equal to $+2.6 \%$ and $-2.0 \%$ for the $0.9 \mathrm{~g}$ and $1.1 \mathrm{~g}$ cases, respectively. Exergy losses via viscous irreversibilities decrease for a steeper slope of the pulse (see Table 4) leading the Bejan number to monotonically increase in the single components of the system (see Fig. 12(b)).

The results highlight the importance of the temporal gradient on the turbine performance and the consequent risk of not considering it in the amplitude and frequency studies. Despite the impact of the temporal gradient is slightly smaller compared to them, the parameters counteract each other in terms of turbine work trends. Indeed, in terms of turbine work, the beneficial effects of increasing the pulse amplitude and frequency are counteracted by the detrimental ones given by the steeper temporal gradient. As a consequence, it is important to decouple the amplitude and

Table 4 Temporal gradient study quantitative data for the time integral of turbine power (term $C$ ), flow exergy loss via heat transfer (term $D$ ), and total internal irreversibilities (term $E$ with reference to Eq. (4)) with viscous and thermal contributions highlighted. Cycle-averaged and maximum temperatures are calculated at the inlet volute section $\left(\mathcal{S}_{1}\right)$. Cycle-averaged and maximum turbine torque are also reported.

\begin{tabular}{|c|c|c|c|c|c|c|c|c|c|}
\hline & \multicolumn{3}{|c|}{ Exergy budget } & \multicolumn{2}{|c|}{ Internal irreversibilities } & \multicolumn{2}{|c|}{ Temperature } & \multicolumn{2}{|c|}{ Torque } \\
\hline & $C(\mathrm{~J})$ & $D(\mathrm{~J})$ & $E(\mathrm{~J})$ & $T_{o} S_{\text {gen,viscous }}(\mathrm{J})$ & $T_{o} S_{\text {gen,thermal }}(\mathrm{J})$ & $T_{\mathcal{S}_{2}, \text { avg }}(\mathrm{K})$ & $T_{\mathcal{S}_{2}, \max }(\mathrm{K})$ & $\tau_{\text {avg }}(\mathrm{Nm})$ & $\tau_{\max }(\mathrm{Nm})$ \\
\hline $0.9 \mathrm{~g}$ & 721 & 279 & 155 & 125 & 30 & 1123 & 1418 & 1.14 & 3.22 \\
\hline Baseline & 696 & 291 & 151 & 120 & 31 & 1138 & 1447 & 1.10 & 3.18 \\
\hline $1.1 \mathrm{~g}$ & 677 & 301 & 148 & 116 & 32 & 1153 & 1455 & 1.07 & 3.15 \\
\hline
\end{tabular}


frequency effects from the temporal gradient ones in order to achieve a clear characterization of the turbine response to the pulse characteristics.

\section{Discussion}

In the present research work, the response of a turbocharger radial turbine to engine-like pulsating flow conditions is analyzed numerically for different amplitudes, frequencies, and pulse temporal gradients, respectively. Since the target is primarily investigating the response of the turbine with respect to the different pulse characteristics, the total temperature profile has been uniformly scaled in order to guarantee a constant cycle-averaged inflow exergy among different cases. This constraint guarantees complete comparability of the different cases: the system performance is not affected by a different exergy content, i.e., the theoretical maximum work achievable. However, this choice has a direct effect on the heat transfer trend expressed in the different exergy budget graphs. Indeed, if the total temperature profile is not scaled, the heat transfer trends are reported reversed in case of the amplitude and temporal gradient studies.

\section{Conclusions}

This work investigates the effects of pulse characteristics on the turbine performance, as well as the impact on heat transfer and entropy generation, in a turbocharger radial turbine under onengine operating conditions. Despite the analysis concerns with pulsating flow conditions, the validation of the model was limited to the comparison between numerical results and experimental data obtained in gas-stand experiments. In order to assess the generality of the model, the entire performance map has been covered by means of steady RANS simulations. However, it is not known how the numerical results are compared under pulsating flow conditions. For the analysis, the mass flow rate pulse has been first parameterized through three parameters: amplitude, frequency, and temporal gradient. Then, each parameter has been varied by considering a $\pm 10 \%$ variation with respect to a baseline pulse, while keeping the others fixed. The major aim of the adopted methodology is to first isolate the effect of the pulse amplitude and frequency to the temporal gradient influence contrary to previous literature studies. In addition, to enable a direct comparison of the importance of the different parameters, the cycle-averaged inflow exergy has been kept constant so that the system performance is not affected by a different theoretical maximum work achievable.

The results presented highlight two main conclusions on the turbine response to pulse shape:

- Increasing pulse amplitude and frequency have beneficial effects on the turbine performance, both in terms of turbine work and efficiency. In the amplitude analysis, the average percentage variation of the turbine work has been reported equal to $6.8 \%$, identifying it as the driving parameter of the turbine performance.

- According to the given definition, increasing the temporal gradient has a detrimental effect in terms of turbine performance and a smaller influence compared to variations of the pulse amplitude.

These two main conclusions highlight a third important aspect. The previous literature studies investigating the amplitude and frequency effects cannot provide a clear characterization of the outcomes, in any case not without careful consideration of initial and boundary conditions. Note that the temporal gradient is characterized by opposite effects as compared to amplitude and frequency, and an increase in both amplitude and frequency is linked to a steeper slope of the pulse and, thus, a change in the temporal gradient. It is therefore important to provide proper boundary conditions able to decouple temporal gradient effects from pulse amplitude and frequency in order to achieve a clear characterization of the turbine response to pulse characteristics. Moreover, each case should be characterized by the same theoretical maximum work achievable so that the trends of the turbine performance are only influenced by the pulse characteristics. In this sense, exergy represents a suitable quantity for such analysis.

The second objective of the performed study is the analysis of the heat transfer and entropy generation mechanisms response to the pulse characteristics in different components of the hot side. The results highlight that

- Increasing the pulse amplitude and frequency decreases the heat transfer in both exhaust manifold and volute, while an increase of the temporal gradient produces an opposite trend.

In particular, for the amplitude and temporal gradient studies, variations of heat transfer are driven by the scaling of the total temperature profile, performed in order to match the cycleaveraged inflow exergy. Auxiliary simulations not published in this paper have otherwise demonstrated that the trend is opposite if the same total temperature profile is imposed as boundary condition for both characteristics. Total internal irreversibilities, as the relative importance between viscous and thermal entropy generation, are reported to experience peculiar trends with respect to variations of the pulse characteristics:

- An increase of the total internal irreversibilities is reported for larger pulse amplitudes and smaller frequencies and temporal gradients.

- Total internal irreversibilities are particularly influenced by the pulse amplitude. The average variation with respect to the pulse amplitude is reported equal to $12 \%$, mainly related to the increase of viscous entropy generation caused by larger velocity gradients.

Smaller variations are otherwise reported in the frequency and temporal gradient studies, where exergy losses via total irreversibilities are subject to a decreasing trend correlated to a reduction of viscous entropy generation.

Based on the results observed, the optimum pulse shape tends to the one characterized by maximum amplitude and minimum temporal gradient and intra-pulse duration.

\section{Acknowledgment}

This study was conducted within the Competence Center for Gas Exchange (CCGEx) at KTH. The authors would like to acknowledge the Swedish Energy Agency and CCGEx's industrial partners for their support and contributions. The support from EU project VISION-xEV, which has received funding from the European Union's Horizon 2020 Research and Innovation Program under Grant Agreement No. 824314 is acknowledged by S.M. Lim and M. Mihaescu. KTH PDC under the Swedish Computing Infrastructure (SNIC) is also acknowledged for providing the computational resources required to perform the simulations.

\section{Funding Data}

- European Union's Horizon 2020 Research and Innovation Program (Grant Agreement No. 824314; Funder ID: $10.13039 / 100010661)$.

$$
\begin{aligned}
& \text { Nomenclature } \\
& \mathrm{Be}=\text { Bejan number } \\
& c_{p}=\text { specific heat at constant pressure, } \mathrm{J} / \mathrm{kg} \mathrm{K} \\
& D=\text { diameter, } \mathrm{m} \\
& e_{f}=\text { specific exergy, } \mathrm{J} / \mathrm{kg} \\
& \mathbf{f}=\text { force per unit area, } \mathrm{N} / \mathrm{m}^{2} \\
& h=\text { specific enthalpy, } \mathrm{J} / \mathrm{kg} \\
& k=\text { thermal conductivity, } \mathrm{W} / \mathrm{m} \mathrm{K} \\
& \dot{m}=\text { mass flow rate, } \mathrm{kg} / \mathrm{s} \\
& \mathrm{MFP}=\text { mass flow parameter, } \mathrm{kg} / \mathrm{s} \sqrt{\mathrm{K}} / \mathrm{Pa}
\end{aligned}
$$




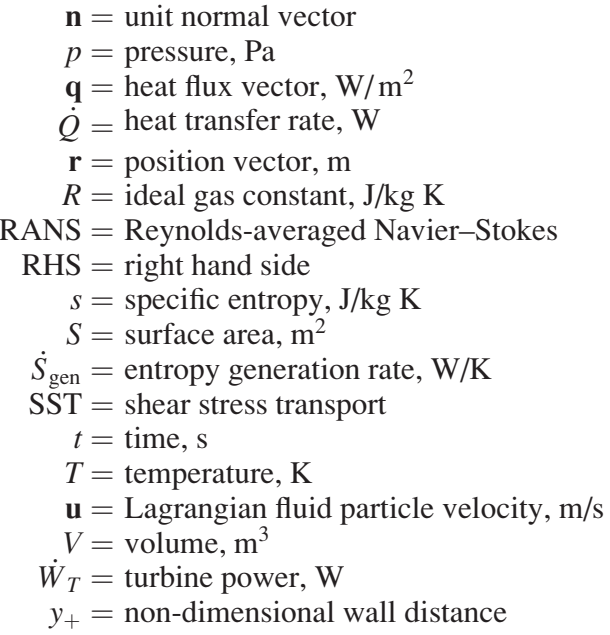

\section{Greek Symbols}

$\eta_{\mathrm{ex}}=$ exergy utilization

$\eta_{\text {is }}=$ isentropic efficiency

$\pi=$ expansion ratio

$\rho=$ density, $\mathrm{kg} / \mathrm{m}^{3}$

$\tau=$ turbine torque, $\mathrm{N} \mathrm{m}$

$\phi=$ generic quantity

$\Lambda=$ lambda criterion

$\mu=$ molecular dynamic viscosity, $\mathrm{Pa} \mathrm{s}$

$\boldsymbol{\Omega}=$ rotational speed vector, $\mathrm{rad} / \mathrm{s}$

\section{Superscripts and Subscripts}

$$
\begin{aligned}
\text { avg } & =\text { average } \\
\text { eff } & =\text { effective } \\
\text { gen } & =\text { generation } \\
\text { in } & =\text { inlet } \\
\operatorname{man} & =\text { manifold } \\
\max & =\text { maximum } \\
o & =\text { dead state } \\
\text { out } & =\text { outlet } \\
t & =\text { total quantity } \\
w & =\text { wall }
\end{aligned}
$$

\section{References}

[1] Copeland, C. D., Martinez-Botas, R., and Seiler, M., 2012, “Unsteady Performance of a Double Entry Turbocharger Turbine With a Comparison to Steady Flow Conditions," ASME J. Turbomach., 134(2), p. 021022.

[2] Wallace, F. J., and Blair, G. P., 1965, "The Pulsating-Flow Performance of Inward Radial-Flow Turbines," ASME Paper No. 65-GTP-21.

[3] Benson, R., and Scrimshaw, K., 1965, "An Experimental Investigation of NonSteady Flow in a Radial Gas Turbine," Proceedings of the Institution of Mechanical Engineers, Conference Proceedings, SAGE Publications: London, UK, 180(10), pp. 74-85.

[4] Benson, R. S., 1974, "Nonsteady Flow in a Turbocharger Nozzleless Radial Gas Turbine," SAE Technical Paper No. 740739.

[5] Kosuge, H., Yamanaka, N., Ariga, I., and Watanabe, I., 1976, "Performance of Radial Flow Turbines Under Pulsating Flow Conditions," ASME J. Eng. Power, 98(1), pp. 53-59.

[6] Capobianco, M., Gambarotta, A., and Cipolla, G., 1989, "Influence of the Pulsating Flow Operation on the Turbine Characteristics of a Small Internal Combustion Engine Turbocharger," IMechE Paper No. C372/019.

[7] Capobianco, M., and Gambarotta, A., 1990, "Unsteady Flow Performance of Turbocharger Radial Turbines," Proceeding of the Institute of Mechanical Engineers, Fourth International Conference of Turbocharging and Turbochargers, London, UK, Paper No. C405/017, pp. 123-132.

[8] Dale, A., and Watson, N., 1986, "Vaneless Radial Turbocharger Turbine Performance," ImechE Conference Transactions; Turbochargers and Turbocharging, London, UK, May 6-8, pp. 65-76.
[9] Szymko, S., Martinez-Botas, R. F., and Pullen, K. R., 2005, "Experimental Evaluation of Turbocharger Turbine Performance Under Pulsating Flow Conditions," ASME Paper No. GT2005-68878.

[10] Marelli, S., and Capobianco, M., 2011, "Steady and Pulsating Flow Efficiency of a Waste-Gated Turbocharger Radial Flow Turbine for Automotive Application," Energy, 36(1), pp. 459-465.

[11] Padzillah, M., Rajoo, S., Yang, M., and Martinez-Botas, R., 2015, "Influence of Pulsating Flow Frequencies Towards the Flow Angle Distributions of an Automotive Turbocharger Mixed-Flow Turbine," Energy Convers. Manag., 98, pp. 449-462.

[12] Galindo, J., Fajardo, P., Navarro, R., and García-Cuevas, L., 2013, "Characterization of a radial turbocharger turbine in Pulsating Flow by Means of CFD and Its Application to Engine Modeling," Appl. Energy, 103, pp. $116-127$.

[13] Cao, T., Xu, L., Yang, M., and Martinez-Botas, R. F., 2014, "Radial Turbine Rotor Response to Pulsating Inlet Flows,” ASME J. Turbomach., 136(7).

[14] Yang, M., Deng, K., Martines-Botas, R., and Zhuge, W., 2016, "An Investigation on Unsteadiness of a Mixed-Flow Turbine Under Pulsating Conditions," Energy Convers. Manag., 110, pp. 51-58.

[15] Ding, Z., Zhuge, W., Zhang, Y., Chen, H., and Martinez-Botas, R., 2017, "Investigation on Pulsating Flow Effect of a Turbocharger Turbine," ASME Paper No. V01AT02A008.

[16] Zhao, R., Li, W., Zhuge, W., Zhang, Y., Yin, Y., and Wu, Y., 2018, "Characterization of Two-Stage Turbine System Under Steady and Pulsating Flow Conditions," Energy, 148, pp. 407-423.

[17] Chen, H., and Winterbone, D., 1990, "A Method to Predict Performance of Vaneless Radial Turbines Under Steady and Unsteady Flow Conditions," IMechE Turbocharging and Turbochargers, Paper No. C405/008, pp. 13-22.

[18] Copeland, C., Newton, P., Martinez-Botas, R., and Seiler, M., 2012, "A Comparison of Timescales Within a Pulsed Flow Turbocharger Turbine," 10th International Conference on Turbochargers and Turbocharging, London, UK, May 15-16, pp. 389-404.

[19] Piscaglia, F., Onorati, A., Marelli, S., and Capobianco, M., 2019, “A Detailed One-Dimensional Model to Predict the Unsteady Behavior of Turbocharger Turbines for Internal Combustion Engine Applications," Int. J. Engine Res., 20(3), pp. 327-349.

[20] Lee, S. P., Rezk, A., Jupp, M., and Nickson, K., 2016, "The Influence of Pulse Shape on the Performance of a Mixed Flow Turbine for Turbocharger Applications," Int. J. Mech. Eng. Rob. Res., 7(2), pp. 136-142.

[21] Rezk, A., Sharma, S., Barrans, S., Hossain, A. K., Lee, P. S., and Imran, M. 2021, "Computational Study of a Radial Flow Turbine Operates Under Various Pulsating Flow Shapes and Amplitudes," ASME J. Energy Resour. Technol., 143(12), pp. 1-24.

[22] Lim, S. M., Dahlkild, A., and Mihaescu, M., 2019, "Influence of Upstream Exhaust Manifold on Pulsatile Turbocharger Turbine Performance," ASME J. Eng. Gas Turbines Power, 141(6), p. 061010.

[23] Menter, F. R., 1994, "Two-Equation Eddy-Viscosity Turbulence Models for Engineering Applications," AIAA J., 32(8), pp. 1598-1605.

[24] Jarrin, N., Benhamadouche, S., Laurence, D., and Prosser, R., 2006, "A Synthetic-Eddy-Method for Generating Inflow Conditions for Large-Eddy Simulations," Int. J. Heat Fluid Flow, 27(4), pp. 585-593.

[25] Hellstrom, F., and Fuchs, L., 2008, "Effects of Inlet Conditions on the Turbine Performance of a Radial Turbine," ASME Paper No. GT2008-51088.

[26] Burke, R., Vagg, C., Chalet, D., and Chesse, P., 2015, "Heat Transfer in Turbocharger Turbines Under Steady, Pulsating and Transient Conditions," Int. J. Heat Fluid Flow, 52, pp. 185-197.

[27] Gao, X., Savic, B., and Baar, R., 2019, "A Numerical Procedure to Model Heat Transfer in Radial Turbines for Automotive Engines," Appl. Therm. Eng., 153, pp. 678-691.

[28] Lim, S. M., Bakhshmand, S. K., Biet, C., and Mihaescu, M., 2020, "Experimental and Numerical Investigation of a Turbocharger Turbine Using Exergy Analysis at Non-Adiabatic Conditions," SAE Technical Paper No. 2020-01-2225.

[29] Lim, S. M., Dahlkild, A., and Mihaescu, M., 2018, "Aerothermodynamics and Exergy Analysis in Radial Turbine With Heat Transfer," ASME J. Turbomach., 140(9), p. 091007.

[30] Lim, S. M., 2018, “Aerothermodynamics and Exergy Analysis in Turbocharger Radial Turbine,” Ph.D. thesis, KTH Royal Institute of Technology, Stockholm, Sweden.

[31] Bejan, A., 2002, "Fundamentals of Exergy Analysis, Entropy Generation Minimization, and the Generation of Flow Architecture," Int. J. Energy Res., 26(7), pp. $0-43$.

[32] Herwig, H., and Kock, F., 2006, "Direct and Indirect Methods of Calculating Entropy Generation Rates in Turbulent Convective Heat Transfer Problems," Heat Mass Transfer, 43(3), pp. 207-215.

[33] Bejan, A., 2013, Entropy Generation Minimization: The Method of Thermodynamic Optimization of Finite-Size Systems and Finite-Time Processes, CRC Press, Boca Raton, FL.

[34] Zhao, R., Li, W., Zhuge, W., and Zhang, Y., 2019, "Unsteady Flow Loss Mechanism and Aerodynamic Improvement of Two-Stage Turbine Under Pulsating Conditions," Entropy, 21(10), p. 985. 